\title{
Local Moment Formation in the Periodic Anderson Model with Superconducting Correlations
}

\author{
M. A. N. Araújo and N. M. R. Peres \\ Departamento de Física, Universidade de Évora, Rua Romão Ramalho, 59, P-7000-671 Évora Codex, Portugal \\ Centro de Física da Universidade do Minho, Campus Gualtar, P-4700-320, Portugal \\ P. D. Sacramento \\ Departamento de Física and CFIF, Instituto Superior Técnico, Av. Rovisco Pais, 1049-001 Lisboa, Portugal
}

(November 16, 2018)

\begin{abstract}
We study local moment formation in the presence of superconducting correlations among the $f$-electrons in the periodic Anderson model. Local moments form if the Coulomb interaction $U>U_{c r}$. We find that $U_{c r}$ is considerably stronger in the presence of superconducting correlations than in the non-superconducting system. Our study is done for various values of the $f$-level energy and electronic density. The smallest critical $U_{c r}$ values occur for the case where the number of $f-$ electrons per site is equal to one. In the presence of $d$-wave superconducting correlations we find that local moment formation presents a quantum phase transition as function of pressure. This quantum phase transition separates a region where local moments and $d$-wave superconductivity coexist from another region characterized by a superconducting ground state with no local moments. We discuss the possible relevance of these results to experimental studies of the competition between magnetic order and superconductivity in $\mathrm{CeCu}_{2} \mathrm{Si}_{2}$.

PACS numbers: 75.20.Hr, 71.27.+a, 74.70.Tx
\end{abstract}

The superconducting and magnetic properties of heavy-fermion materials have attracted much attention mainly due to their non-conventional character.1. All these materials have very large specific heat coefficients $\gamma$, indicating very large effective masses, hence the designation heavy fermions. The complexity of these systems arises from the interplay between Kondo screening of local moments, the antiferromagnetic (RKKY) interaction between the moments and superconducting correlations between the heavy quasi-particles. On the experimental side, these systems exhibit phases in which antiferromagnetic ordering of the local moments may coexist with unconventional superconductivity, and/or phases with no magnetic ordering. The latter could be either due to Kondo screening of the local moments or to a spin liquid arrangement of the local moments.

For systems that exhibit both superconductivity and antiferromagnetism, usually $U$-based heavy-fermions, the ratio between the Néel temperature $T_{N}$ and the superconducting critical temperature $T_{c}$ is of the order of $T_{N} / T_{c} \sim 1-10$, with coexistence of both types of order below $T_{c}$. The coexistence of both types of order can be tuned by external parameters such as external pressure or changes in the stoichiometry. Exit Examples of heavyfermion materials which exhibit antiferromagnetic and superconducting order at low temperature are $U R u_{2} S i_{2}$ and $U_{0.97} T h_{0.03} B e_{13}$. It has recently been found that $U P d_{2} A l_{3}\left(T_{N}=14.3 \mathrm{~K}\right.$ and $\left.T_{c}=2 \mathrm{~K}\right)$ and $U N i_{2} A l_{3}$ $\left(T_{N}=4.5 \mathrm{~K}\right.$ and $\left.T_{c}=1.2 \mathrm{~K}\right)$ show coexistence of super conductivity and local moment antiferromagnetism. In the $C e$-based heavy-fermions however typically magnetism competes with superconductivity. In the proto- type heavy-fermion system $\mathrm{Ce}_{x} \mathrm{Cu}_{2} \mathrm{Si}_{2}$ the competition of $d$-wave superconductivity and magnetic order was clearly identified in a small range of $x$ values around $x \simeq 0.99$.

A description of the normal state properties of the heavy-fermion systems has been attempted assuming a generalifation of the impurity Anderson model to the lattice. 10 In the Anderson lattice the energy of a single electron in an $f$-orbital (e. g. $4 f^{1}$ ) is $\epsilon_{0}$, and the energy of two electrons in the same $f$-orbital $\left(4 f^{2}\right)$ is $2 \epsilon_{0}+U$, where $U$ is the on-site Coulomb repulsion. The energy of the $4 f^{2}$ state is much larger than the energy of the $4 f^{1}$ state. In the case of interest the empty $f$-orbital lies below the Fermi level $\epsilon_{F}$, whereas the doubly occupied $f$-orbital has higher energy than $\epsilon_{F}$. Since in many systems $\epsilon_{0}+U \gg \epsilon_{F}$ the simplifying limit $U \rightarrow \infty$ is taken is some theoretical studies.

The limit $U_{-}=\infty$ has been studied using the slave boson technique.10 13 In particular, it has been shown that superconducting instabilities arise in the $p$ and $d$-wave channels because of the effective (RKKY) interaction between the $f$-electrons. 1214 Mean-field studies of superconductivity in the Anderson lattice 2 th at finite $U$ and at $U=\infty$ have recently been done 13,15 Nevertheless, the coexistence of superconducting correlations and magnetic ordering of local moments in heavy-fermion systems has not yet, to our knowledge, been studied theoretically.

In this work we aim to establish the conditions for the appearance of local moments in the Anderson lattice when superconducting correlations among the $f$-electrons are also present. In order to decide whether a $f$-site behaves as a local moment we use a criterion 
identical to that introduced by Anderson for the single impurity problem16, which we shall now briefly review. The procedure in Ref 16 consists of making a Hartree-Fock decoupling of the interaction at the impurity ( $f$ site):

$$
U \hat{n}_{\uparrow}^{f} \hat{n}_{\downarrow}^{f} \rightarrow U\left\langle\hat{n}_{\uparrow}^{f}\right\rangle \hat{n}_{\downarrow}^{f}+U \hat{n}_{\uparrow}^{f}\left\langle\hat{n}_{\downarrow}^{f}\right\rangle-U\left\langle\hat{n}_{\uparrow}^{f}\right\rangle\left\langle\hat{n}_{\downarrow}^{f}\right\rangle,
$$

where $\hat{n}_{\uparrow}^{f}, \hat{n}_{\downarrow}^{f}$ are the number operators at the $f$ site and their expectation values $\left\langle\hat{n}_{\uparrow}^{f}\right\rangle,\left\langle\hat{n}_{\downarrow}^{f}\right\rangle$ must be found self-consistently. Then, the symmetry beaking solutions with $m=\left\langle\hat{n}_{\uparrow}^{f}\right\rangle-\left\langle\hat{n}_{\downarrow}^{f}\right\rangle \neq 0$ correspond to the local moment regime. Therefore, the local moments arise as the symmetry-breaking local minima, with $\left\langle\hat{n}_{\uparrow}^{f}\right\rangle \neq\left\langle\hat{n}_{\downarrow}^{f}\right\rangle$, of the effective action for the impurity in a Hartree-Fock decoupling scheme. Such a mean-field treatment (11) does not account for the dynamics of the local moment which arises from its effective interaction with the conduction electrons.

In this work we propose to extend such ideas to the lattice of $f$-sites taking phenomenologically into account the presence of superconducting correlations between the $f$-electrons. Because we consider a lattice of $f$ sites, we have to look for mean-field solutions with some previously chosen spatial arrangement of the moments which will be taken as either ferromagnetic or antiferromagnetic. Since heavy fermion systems tend to exhibit antiferromagnetic order, we have chosen this type of broken symmetry state. Some comments are also made on the ferromagnetic case. We recall that this mean-field treatment leaves out the dynamics of the local moments. Such dynamics lies beyond the scope of this work but would possibly allow to describe the nature of the magnetic phases of the system including Kondo screening, or ordering due to RKKY interaction, or a spin liquid arrangement of the local moments, etc.

Our study of the interplay between superconducting correlations and local moment formation may be relevant to the understanding of recent studies of $\mathrm{CeCu}_{2} \mathrm{Si}_{2}$ samples near stoichiometric composition, where it has been observed that a $d$-wave superconducting phase-expels a magnetic "A phase" under increasing pressure 17.18.

We wish to investigate the appearance of local moments in the Anderson lattice in the presence of superconducting correlations. Because a microscopic description of superconductivity in heavy-fermion systems is still lacking we opt to treat superconductivity by explicitly adding a phenomenological pairing term $H_{J}$ to the Anderson lattice Hamiltonian for spin 1/2 electrons:

$$
H=H_{0}+H_{U}+H_{J}
$$

where

$$
\begin{aligned}
H_{0} & =\sum_{\vec{k}, \sigma}\left(\epsilon_{\vec{k}}-\mu\right) c_{\vec{k}, \sigma}^{\dagger} c_{\vec{k}, \sigma}+\sum_{i, \sigma}\left(\epsilon_{0}-\mu\right) f_{i, \sigma}^{\dagger} f_{i, \sigma} \\
& +V \sum_{i, \sigma}\left(c_{i, \sigma}^{\dagger} f_{i, \sigma}+f_{i, \sigma}^{\dagger} c_{i, \sigma}\right)
\end{aligned}
$$

$$
\begin{aligned}
H_{U} & =U \sum_{i} \hat{n}_{i, \uparrow} \hat{n}_{i, \downarrow}, \\
H_{J} & =J \sum_{\vec{k}}\left(f_{\vec{k}, \uparrow}^{\dagger} f_{-\vec{k}, \downarrow}^{\dagger} \Delta(\vec{k})+\text { H.c. }\right),
\end{aligned}
$$

where $\epsilon_{\vec{k}}$ is the dispersion of the $c$-electrons, $\epsilon_{0}$ is the bare energy of the localized $f$-states, $V$ is the hybridization matrix element (assumed $\vec{k}$ independent), $U$ is the on-site Coulomb interaction, and $\hat{n}_{i, \sigma}=$ $f_{i, \sigma}^{\dagger} f_{i, \sigma}$. The gap function $\Delta(\vec{k})$ can be written as $\eta(\vec{k}) \Delta$, where $\eta(\vec{k})$ indicates the pairing symmetry and $\Delta=\frac{1}{N_{s}} \sum_{\vec{k}}\left\langle f_{-\vec{k}, \downarrow} f_{\vec{k}, \uparrow}\right\rangle \eta_{\vec{k}}$. To simplify the calculations we consider a two dimensional system. The results for three dimensions are similar. In 2D we have $\eta_{\vec{k}}^{(s)}=\cos \left(k_{x}\right)+$ $\cos \left(k_{y}\right), \eta_{\vec{k}}^{(p, i)}=\sqrt{2} \sin \left(k_{i}\right), \eta_{\vec{k}}^{(d)}=\cos \left(k_{x}\right)-\cos \left(k_{y}\right)$, for $s, p$, and $d$ wave, respectively.

The local moment formation is investigated by applying the decoupling (1) to the term $H_{U}$. We seek meanfield solutions such that the electron occupation at the $f$-site $i$ is given by

$$
\left\langle f_{i, \sigma}^{\dagger} f_{i, \sigma}\right\rangle=\frac{1}{2} n_{f}+\frac{1}{2} \sigma m \cos \left(\vec{R}_{i} \cdot \vec{Q}\right),
$$

where $n_{f}$ and $\vec{R}_{i}$ denote the mean number of $f$ electrons and the position of the $i$ lattice site, respectively, and $\sigma= \pm 1$. This choice corresponds to a spatially periodic arrangement of the local moments where $\vec{Q}$ is the ordering vector. Since we shall perform the calculation on a 2D square lattice the choice $\vec{Q}=0$ gives ferromagnetic order and the choice $\vec{Q}=(\pi, \pi)$ gives antiferromagnetic order.

The mean-field parameters $m, n_{f}=n_{\uparrow}^{f}+n_{\downarrow}^{f}$, and $\Delta$ are obtained from the minimization of the free energy. The chemical potential is determined imposing total particle number conservation $n=n_{c}+n_{f}$. We perform the calculations for a conduction band dispersion of form: $\epsilon_{\vec{k}}=-2 t \sum_{i=x, y} \cos \left(k_{i}\right)$. All the energies and temperatures in this work are measured in units of the hopping integral $t$, and we have set $t=1$. The possible pairing symmetries have been studied separately.

For a given set of parameters $n, \epsilon_{0}, V$, and $J$ one may ask what is the minimum value of the local repulsion, $U_{c r}$, for which ground-state solutions with $(\Delta, m) \neq 0$ appear. Figure 11 shows $1 / U_{c r}$ for each $\epsilon_{0}$ and two electronic densities. The local moment regime appears for $U>U_{c r}$. For comparison we also show $U_{c r}$ for a nonsuperconducting system $(J=0)$. It is seen from Figure 11 that the appearance of local moments in a superconducting system requires considerably stronger $U$ than in a normal system. For each curve the $f$ level occupancy $n_{f}$ increases upon increasing $\epsilon_{0}$ and the values of $\epsilon_{0}$ at which $U_{c r}$ is minimum (left panel of Figure 1) correspond to $n_{f}=1$. Therefore we also see that smaller $U_{c r}$ occurs when $f$ level occupancy is close to one. If $n_{f}<1$ then $U$ can be increased without destroying superconductivity 
but if $n_{f}>1$ then ingreasing $U$ will eventually destroy superconductivity. 13,15 We note that the relative position of the superconducting curves in the two panels of Figure 11 is different.
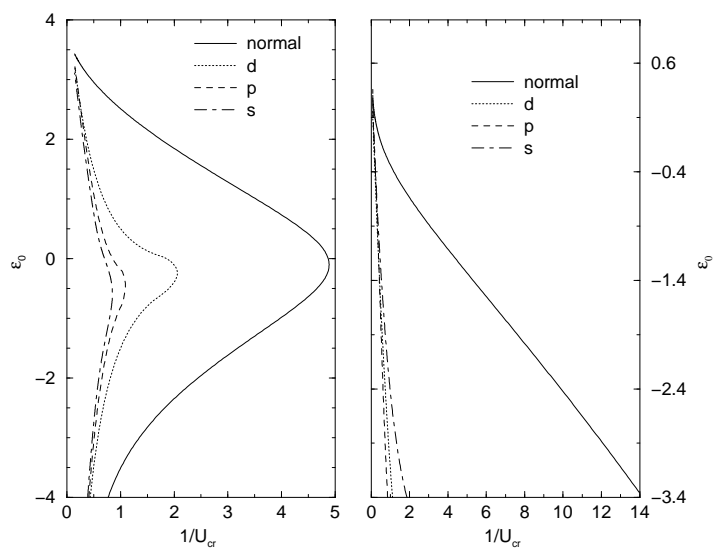

FIG. 1. Relation between the critical $U_{c r}$ and the $f$ level bare energy $\epsilon_{0}$, for the parameters $J=-1.7, V=0.4, t=1$, and: left panel: $n=2$; right panel: $n=1$. The curve for the normal system corresponds to $J=0$. Calculations were performed for temperature $T=10^{-3}$.

We have also considered the case of a ferromagnetic arrangement of the local moments $(Q=0)$. Our calculations show that, for the same parameters, ferromagnetic solutions require much stronger $U$ values $\left(1 / U_{c r} \leq 0.16\right)$ even in the absence of superconductivity. This seems to be an indication that, in a lattice, there is a tendency for local moments to form in an antiferromagnetic arrangement.

The dependence of the Néel and superconducting temperatures on pressure has been measured in some heavyfermion systems. 3 In those studies the Néel temperature is found to decrease as the applied pressure increases and superconducting order is found to develop in a limited range of applied pressures when the Néel temperature is reduced below $\sim 1 \mathrm{~K}$.

It is interesting to see how the mean field critical temperatures in our model vary with the model parameters which, in principle, should depend on externally appplied pressure. Increasing pressure should, presumably, make the hybridization $V$ and the conduction band hopping $t$ increase while keeping $U$ and possibly $J$ almost constant.20 In Figure 2 we plot the mean field critical temperatures as function of $V$ while keeping the ratio $V / t$ fixed. An interesting feature occurs for the $d$-wave pairing symmetry: if the temperature $T^{*}$ that marks the onset of $m \neq 0$ solutions is higher than the superconducting temperature $T_{c}$ then $d$-wave superconductivity and local moments coexist at low temperature; but once $T_{c}$ becomes greater than $T^{*}$ the latter drops abruptly and we cannot find magnetic solutions at the lowest temperatures. At zero temperature we have a quantum phase transition that can be tuned using the external pressure: as pressure is reduced (at zero temperature) the ground state nature of the system changes from non-magnetic but superconducting to magnetic and superconducting at the critical value $V_{c} / \epsilon_{0} \sim 0.38$. We find this transition to be of first order. Such behaviour is not observed for $s$ - or $p$-wave symmetries.

Going beyond mean field, and in general terms, the local moments due to the $f$-electrons are progressively quenched as the temperature lowers. In dilute systems the picture is well understood as due to the Kondo screening by the conduction electrons. In dense systems however the picture is more involved. At low temperatures the local moments are not completely quenched. For several $U$-based materials like $U R u_{2} S_{2}, U P t_{3}$ or $U P d_{3}$ the remaining moments are quite small of the order of $0.01-0.03 \mu_{B}$ but for other systems like $U P d_{2} A l_{3}$ the local moment is quite large of the order of $0.85 \mu_{B}$. A reason is the Kondo-Nozières compensation theorem that states that in the lattice there are not enough available conduction electrons to quench the local moments.

The $\mathrm{Ce}$-based compounds like $\mathrm{CeCu}_{2} \mathrm{Si}_{2}$ and $\mathrm{CeCu}_{6} \mathrm{Au}$ show competition between magnetism and superconductivity if doped and/or under pressure with a phase higher in temperature where spin fluctuations are significant. The behavior of these systems has been interpretef as due to the vicinity to a fuantum critical point21. Two pictures arise however22: in the first one the Kondo temperature is high (the moments are quenched at a finite temperature) and when the system approaches the quantum critical point there are no free moments (assuming that quenching is complete). Then the system has to order due to a Fermi surface instability of the spin density wave type. Another possible situation is one in which the moments are not completely quenched down to $T=0$ and are free to orient themselves leading to magnetism. In the case of $C e C u_{6-x} A u_{x}$ it has been recently found that the second picture holds22. On the other hand the high value of the Kondo temperature for the $\mathrm{CeCu} \mathrm{Si}_{2}$ compound indicates possibly that the first scenario should hold. Our results show that $d$-wave pairing excludes magnetism through a quantum phase transition. The treatment of a spin-densitywave would be mathematically similar and also lead to the exclusion of the spin-density-wave by $d$-wave pairing as pressure increases. This means that our results might be of relevance for understanding the interplay between superconductivity and magnetic order in the $d$-wave heavy-fermion compound $\mathrm{CeCu}_{2} \mathrm{Si}_{2}$. This system exhibits a magnetic "A phase" at low temperature whose detailed nature is not yet known. Increasing pressure reduces the critical temperature $T_{A}$ of the A phase. Recent studiest 1719 of $\mathrm{CeCu}_{2} \mathrm{Si}_{2}$ samples near stoichiometric composition have shown that a $d$-wave superconducting phase expels the magnetic "A phase" when $T_{A}$ approaches $T_{c}$ under increasing pressure. Our results in Figure 2 show that $d$-wave superconductivity destroys the local moments when $T^{*}$ meets $T_{c}$ as function of increasing hybridization. 


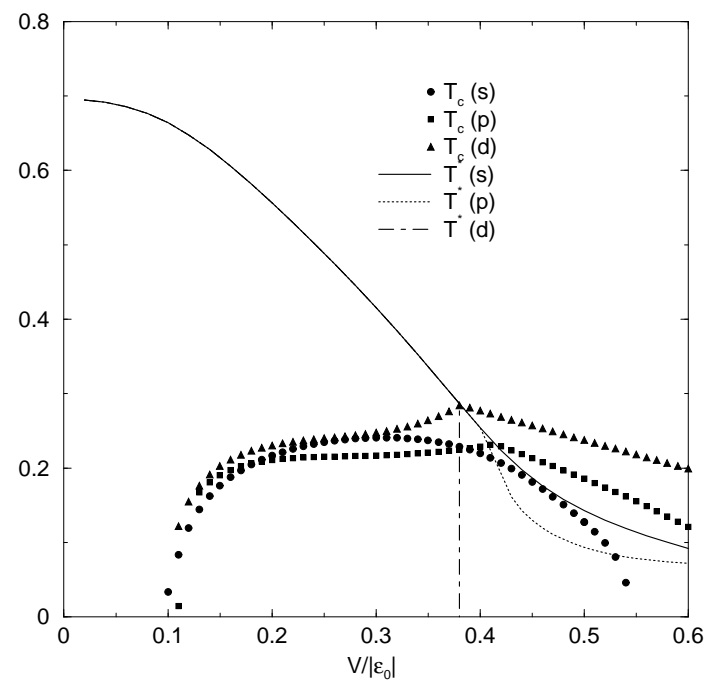

FIG. 2. Mean field magnetic $\left(T^{*}\right)$ and superconducting $\left(T_{c}\right)$ critical temperatures versus $V /\left|\epsilon_{0}\right|$, for a constant ratio $V / t=0.5$. The parameters are $U=2.8, J=-1.7$, $\epsilon_{0}=-3.4$, and $n=1$. The various curves for the magnetic transition coincide up to $V / \epsilon_{0} \sim 0.38$.

In summary, we have studied the formation of local moments in the periodic Anderson model in the presence of superconducting correlations. We identify the local moment regime with the symmetry-breaking saddle-point of the effective action for the $f$-sites in a Hartree-Fock decoupling of local Coulomb repulsion. We have found that local moments tend to order antiferromagnetically. We note that this does not imply that antiferromagnetic order arises in a Slater scenario. Magnetic order in heavy fermion systems is actually believed to be due to the effective RKKY interaction between local moments. This means that once we know the region in parameter space where local moments form, further studies of magnetic order, Kondo screening, etc, possibly require aneffective interaction Hamiltonian for the local moments.23 By simulating the effect of increasing pressure, we have found that d-wave superconductivity competes (rather than coexists) with local moment formation at low temperature above some critical pressure.

This research was supported by Portuguese program PRAXIS XXI under grant number 2/2.1/FIS/302/94.

${ }^{1}$ C. M. Varma, Comments Solid State Physics 11, 221 (1985); Z. Fisk et al., Science 239, 33 (1988); P. Schlottmann, Phys. Reports 181, 1 (1989); Robert H. Heffner and Michael R. Norman, Comments Condensed Matter Physics 17, 361 (1996).

${ }^{2}$ Ronald J. Keizer, Evolution of Magnetism and its Interplay with Superconductivity in Heavy-Fermion $\mathrm{U}(\mathrm{Pt}, \mathrm{Pd})_{3}$,
Ph. D. Thesis, University of Amsterdam, Van der WaalsZeeman Intitute, 1999; Pedro Estrela, Non-Fermi Liquid Behaviour in Uranium-Based Heavy-Fermion Compounds, $\mathrm{Ph}$. D. Thesis, University of Amsterdam, Van der WaalsZeeman Intitute, 2000.

${ }^{3}$ N. D. Mathur, F. M. Grosche, S. R. Julian, I. R. Walker, D. Freye, R. Haselwimmer, and G. Lonzarich, Science 394, 39 (1998).

${ }^{4}$ K. Ishida, Y. Kawasaki, K. Tabuchi, K. Kashima, Y. Kitaoka, K. Asayama, C. Geilbel, and F. Steglich Phys. Rev. Lett 82, 5353 (1999).

${ }^{5}$ R. Caspary, P. Hellmann, M. Keller, G. Sparn, C. Wassilew, R. Köhler, C. Geibel, C. Schank, F. Steglich, and N. Phillips, Phys. Rev. Lett. 71, 2146, (1993).

${ }^{6}$ R. Feyerherm, A. Amato, F. N. Gygax, A. Schenck, C. Geibel, F. Steglich, N. Sato, and T. Komatsubara, Phys. Rev. Lett. 73, 1850 (1994).

${ }^{7}$ N. Bernhoeft, N. Sato, B. Roessli, N. Aso, A. Hiess, G. H. Lander, Y. Endoh, and T. Komatsubara, Phys. Rev. Lett. 81, 4244 (1998);

${ }^{8}$ N. Aso, B. Roessli, N. Bernhoeft, R. Calemczac, N.K. Sato, Y. Endoh, T. Komatsubara, A. Hien, G.H. Lander, H. Kadowaki, Phys. Rev. B 61, R11867 (2000).

${ }^{9}$ D. M. Newns and N. Read, Adv. Phys. 36, 799 (1987).

${ }^{10}$ A.J. Millis and P.A. Lee, Phys. Rev B 35, 3394 (1987).

${ }^{11}$ P. Coleman, Phys. Rev B 29, 3035 (1984); Phys. Rev B 35, 5072 (1987).

12 A. Houghton, N. Read, and H. Won, Phys. Rev. B 37, 3782 (1988).

${ }^{13}$ M.A.N. Araújo, N.M.R. Peres, P.D. Sacramento, and V.R. Vieira, Phys. Rev. B 62, 9800 (2000).

${ }^{14}$ M. Lavagna, A.J. Millis, P.A. Lee, Phys. Rev. Lett. 58, 266 (1987).

15 S. Robaszkiewicz, R. Micnas, and J. Ranninger, Phys. Rev. B 36, 180 (1987); A. Romano, C. Noce, and R. Micnas, Phys. Rev B 55, 12640 (1997); Acta Physica Polonica A 91, 381 (1997).

${ }^{16}$ P. W. Anderson, Phys. Rev. 124, 41 (1961)

17 P. Gegenwart, C. Langhammer, C. Geibel, R. Helfrich, M. Lang, G. Sparn and F. Stelich, Phys. Rev. Lett. 81, 1501 (1998)

${ }^{18}$ G. Bruls, B. Wolf, D. Fisterbush, P. Thalmeier, I. Kouroudis, W. Sun, W. Assmus, B. Lüthi, M. Lang, K. Gloos, F. Steglich and R. Modler, Phys. Rev Lett. 72, 1754 (1994)

${ }^{19}$ G. M. Luke, A. Keren, K. Kojima, L. P. Le, B. J. Sternlieb, W. D. Wu, and Y. J. Uemura, Phys. Rev Lett. 73, 1853 (1994)

${ }^{20}$ Ben Hur Bernhard and Claudine Lacroix, Phys. Rev. B 60, 12149 (1999).

${ }^{21}$ F. Steglich, B. Buschinger, P. Gegenwart, M. Lohmann, R. Helfrich, C. Langhammer, P. Hellmann, L. Donnevert, S. Thomas, A. Link, C. Geibel, M. Lang, G. Sparn and W. Assmus, J. Phys. Cond. Matt. 8, 9909 (1996); H. von Löhneysen, J. Phys. Cond. Matt. 8, 9689 (1996).

${ }^{22}$ A. Schrröder, G. Aeppli, R. Coldea, M. Adams, O. Stockert, H. v. Löhneysen, E. Bucher, R. Ramazashvili and P. Coleman, Nature 407, 351 (2000).

${ }^{23}$ M.A.N Araújo, N.M.R. Peres, and P.D. Sacramento, in preparation. 